\title{
Slippery Thermals and the Cumulus Entrainment Paradox*
}

\author{
Steven C. Sherwood, Daniel HernándeZ-Deckers, And Maxime Colin \\ Climate Change Research Centre, and ARC Centre of Excellence for Climate System Science, \\ University of New South Wales, Sydney, New South Wales, Australia \\ FRANCIS ROBINSON \\ Department of Geology and Geophysics, Yale University, New Haven, Connecticut
}

(Manuscript received 1 August 2012, in final form 19 February 2013)

\begin{abstract}
In numerical simulations of growing congestus clouds, the maximum upward velocities $w$ typically occur in compact toroidal vortices or thermals. These maxima were tracked, and the momentum budget was analyzed within spherical regions centered on them with objectively determined radii approximately enclosing the vortex ring or pair. Such regions are proposed as an advantageous prototype for rising air parcels due to their prolonged identity as evident in laboratory flows. Buoyancy and other forces are generally less than $0.02 \mathrm{~m} \mathrm{~s}^{-2}$ $(0.7 \mathrm{~K})$. In particular, resolved mixing between thermals and their environment fails to produce the drag normally anticipated, often producing even a slight upward force, indicating that parcel models should allow for significantly different dilution rates for momentum than for material properties. A conceptual model is proposed to explain this as a result of the thermals' internal circulation and detrainment characteristics.

The implications of momentum dilution for cumulus development are explored using a simple model of a heterogeneous entraining parcel. Without friction, parcels reach the upper troposphere even at a high entrainment rate $\left[\sim(2 \mathrm{~km})^{-1}\right]$ if the environment is sufficiently humid, whereas with standard momentum dilution, a much lower entrainment rate is required. Peak condensed water amounts and sensitivities of cloud amount and height to ambient humidity are significantly more realistic in the high-entrainment case. This suggests that revised treatments of friction and momentum could help address the "entrainment paradox" whereby entrainment rates implied by detailed cloud studies are higher than those typically preferred for parcel-based calculations.
\end{abstract}

\section{Introduction}

Clouds and deep convection in climate models, predicted based on simple models of the phenomenon, exhibit certain typical and stubborn problems. For one, many models produce too much cloud within a few kilometers of the tropopause, and most produce too little at lower levels (Zhang et al. 2010; Chepfer et al. 2008). They also intrinsically predict the lofting of very high ice amounts to the tallest cloud tops, such that "precipitation efficiencies" approaching 99\% or more (Emanuel 1991)

\footnotetext{
* Supplemental information related to this paper is available at the Journals Online website.
}

Corresponding author address: S. Sherwood, Climate Change Research Centre, Level 4 Matthews Bldg., University of New South Wales, Sydney NSW 2052, Australia.

E-mail: s.sherwood@unsw.edu.au are required to return cloud ice contents to realistic values. This is hard to justify, and resulting ice amounts vary by an order of magnitude among different models (John and Soden 2006). Too much aerosol is also lofted far above the surface in deep convective regions (Winker et al. 2010). Finally, deep convection occurs too early over land, and is simulated almost as readily in environments that are dry aloft as those that are humid, in strong contrast to observations and cloud-resolving model (CRM) simulations indicating that deep growth into environments below $70 \%-80 \%$ relative humidity is nearly impossible in low-shear situations (see Derbyshire et al. 2004; Sherwood et al. 2010).

A number of explanations for these problems are possible, and several strategies have been tried very recently to try to address them (Del Genio 2012). The most popular has been to increase, or make state dependent, the rate of entrainment of environmental air into upward-moving air parcels in deep convection. This 
tends to reduce problems of "overactive" convection and improves intraseasonal variability, but at the expense of degrading the mean state or creating other problems (e.g., Zhou et al. 2012; Mapes and Neale 2011).

Indeed, investigators have been unable to find candidate entrainment profiles that simultaneously produce realistic cloud height, water content, tracer, and other characteristics in standard parcel calculations (Warner 1970; Betts 1982; Simpson 1983; Romps 2010). Some problems can be resolved for the shallow convective case if there is strong entrainment of air from above cloud top, such as the "shedding thermal" (Blyth et al. 1988), but other studies show entrainment at all levels rather than just at cloud top (e.g., Heus et al. 2008). We argue, however, that these ideas fail to explain how such highly entraining cumulus drafts can develop into deep convection.

Observations since the 1950s have implied high entrainment rates also for deep convection (Malkus et al. 1953), and CRMs now suggest typical entrainment rates of order $(2 \mathrm{~km})^{-1}$ throughout the troposphere (Khairoutdinov and Randall 2006; Fridlind et al. 2004), calling into question whether undilute air is ever transported from the surface to the upper troposphere outside of tropical cyclones (Romps and Kuang 2010). Yet diagnostic models of entrainment and schemes in climate models usually predict weak entrainment rates above the first kilometer or two above cloud base (Chikira and Sugiyama 2010), transporting far more undilute air to the upper troposphere (Romps and Kuang 2010). This has seemed necessary to explain the observation that equivalent potential temperature in the tropical upper troposphere is similar to that near the surface (Riehl and Malkus 1958). The additional latent heat released by the freezing of liquid droplets helps to explain this, but it is still difficult for highly entraining parcels to reach the tropopause (Fierro et al. 2009; Romps and Kuang 2010). Diagnostic studies based on standard parcel models often find that entrainment rates decreasing as $z^{-1}$ produce the best agreement with observations (e.g., Holloway and Neelin 2009), but this inference relies on the structural assumptions of the parcel models used, in which entrainment is a tunable parameter.

To fully resolve the above problems may require revising certain founding assumptions of parcel-based convection models. Perhaps the most fundamental issue is how best to represent the basic kinematic structure of a convective cloud. The most favored model has long been a spreading plume (Stommel 1947; Malkus 1954), which is often further idealized as self-similar and statistically uniform in the horizontal (Morton et al. 1956), inspired largely by laboratory observations of neutrally buoyant spreading jets. However, in situ observations and simulations of shallow cumuli often show that cloud motions are dominated by sequences of coherent thermals or warm bubbles (Scorer and Ludlam 1953; Saunders 1961; Carpenter et al. 1998; Zhao and Austin 2005) rather than statistically stationary turbulence. Indeed, cumulus clouds were recognized even in the 1970s as a poor candidate for treatment by theories based on neutrally buoyant jets (Turner 1973,186-196) as they continuously (and inhomogeneously) generate internal heating through condensation, which strongly affects their turbulent characteristics (see Narasimha et al. 2011). Nonetheless, most deep convective schemes, and some shallow ones, begin by treating cumulus clouds as plumes. Deep convective schemes have typically set detrainment during ascent to be much less than entrainment, although numerical studies of shallow convection find that detrainment often balances or can even exceed entrainment (e.g., Siebesma and Cuijpers 1995; Heus et al. 2008).

One of the difficulties when using numerical simulations to diagnose governing parameters of convection is defining exactly what regions should be taken as equivalent to the "rising parcel" invoked in a model or parameterization. Although sophisticated and precise methods for calculating entrainment can be found in the literature (e.g., Romps 2010; Dawe and Austin 2011), the results may be sensitive to this definition, which is fundamentally ambiguous. Cumulus drafts are commonly identified on the basis of cloud water content, buoyancy, and/or vertical velocity criteria (e.g., de Roode and Bretherton 2003). However, none of these characteristics is conservative, which is a fundamental problem if results are to be applied to a Lagrangian parcel calculation. If a rising parcel entrains dry environmental fluid, for example, it cannot remain fully cloudy unless internal mixing is infinitely more rapid than entrainment, which is not consistent with laboratory observations. Nor is the entire parcel necessarily moving upward at all times. On the other hand, environmental air can acquire vertical velocity and, if lifted to its condensation level, become cloudy. While it is possible to overcome some of these ambiguities using tracer methods to calculate entrainment (e.g., Romps 2010), it is not obvious how to use that strategy to diagnose terms in the energy or momentum budgets.

Here we propose a new and, we argue, more dynamically and kinematically consistent definition of what may be taken to constitute a rising, cloudy air parcel, based on the old concept of the quasi-spherical thermal. Despite its simplicity this appears to be robust, and we use it to identify and track thermals in simulated growing cumulus clouds. This allows us to carry out a vertical 
momentum budget on the rising thermals, which suggests that net buoyancy forces and, in particular, drag from in-mixing of stagnant air may be smaller than implied by traditional approaches or assumed in many parcel models. Finally, we show that the behavior of an idealized rising parcel is quite sensitive to friction and that, if the internal heterogeneity of the thermal is considered, one may successfully compute quantities unavailable in traditional calculations.

\section{Explicit cloud simulations}

\section{a. Model configurations}

To begin this study we examined convective clouds growing in a typical tropical environment, as simulated explicitly by version 3.1 of the Weather Research and Forecasting Model (WRF) created by the National Center for Atmospheric Research. While these simulations are surely not perfect representations of real clouds, they do reproduce key aspects of observations (Robinson et al. 2011) and serve here to test the assumptions made in much more idealized cloud models. We focus on the early behavior as cumuli first penetrate the troposphere and form rain (termed here the "congestus" phase), but before they organize and strengthen into a storm.

To simulate individual deep convective events we conducted 24-h runs of the WRF CRM with prescribed initial and boundary conditions. Two 2D and two 3D computational domains were tested, each having a rigid model top at $25 \mathrm{~km}$ with 100 -m vertical spacing, Rayleigh damping in the uppermost $6 \mathrm{~km}$, a free-slip surface, and open boundaries in the $x$ direction. Coarse 2D runs spanned $600 \mathrm{~km}$ in the $x$ direction with 500-m horizontal spacing, while fine ones were conducted with a $150-\mathrm{km}$ domain and $125-\mathrm{m}$ spacing. Coarse 3D runs occupied a $64 \times 600$ domain with $1-\mathrm{km}$ spacing, while fine $3 \mathrm{D}$ runs occupied a $16 \times 150 \mathrm{~km}^{2}$ domain with 250 -m spacing. In the $3 \mathrm{D}$ runs, the $y$ boundary condition was periodic. The narrow domain in the $y$ direction was insufficient to allow full development of 3D mesoscale organization, but was sufficient for convective drafts to be fully threedimensional. The 3D runs also included seed perturbations to initiate small-scale convection.

These simulations are described more fully by Robinson et al. (2011), who show that they reproduce key aspects of satellite observations. In particular, the coarse simulations noted above are those described in that study, augmented here by the higher-resolution ones. While this is not an exhaustive test of resolution dependence, other studies such as that of Romps and Kuang (2010) have shown that entrainment rates at these resolutions are approximately maintained as resolution is further

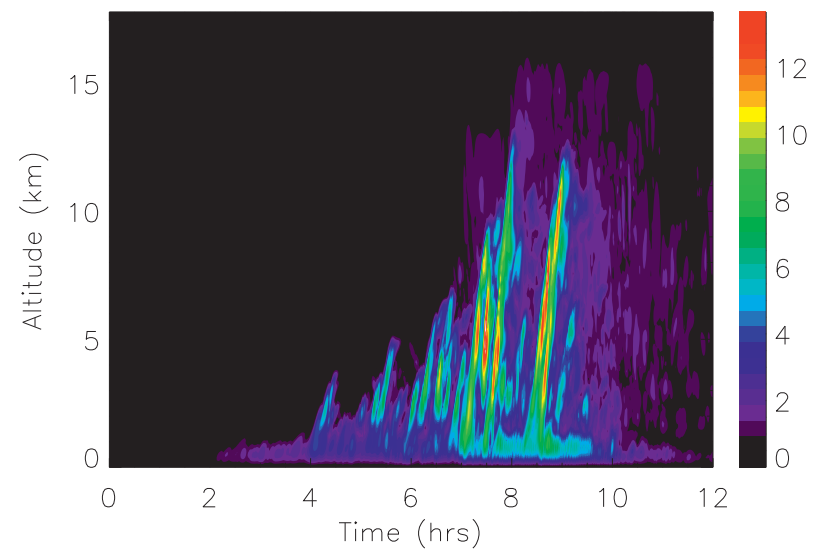

FIG. 1. Maximum upward velocity $\left(\mathrm{m} \mathrm{s}^{-1}\right)$ in the domain at each time and altitude, from the 2DLR simulation.

increased. Physical parameterizations used were the Thompson ice microphysics scheme and the turbulent kinetic energy scheme for subgrid mixing; the radiation and boundary layer schemes were deactivated.

To incite convection, diurnally varying heating was applied to the central portion of the surface over a specified horizontal extent. Several initial conditions of temperature, humidity, and wind were used based on observed tropical soundings. The results shown below are from the simulation with a heated region of $80 \mathrm{~km}$ and the initial sounding denoted HS01 by Robinson et al.; this sounding featured moderate wind shear and typical humidity for the Indo-Pacific region and produced storm intensity characteristics that were about average among those of the various soundings tested in that study.

To test the robustness of results beyond the above changes to resolution, we considered a range of scales of surface heating and an alternate ice microphysical scheme (the Morrison two-moment scheme). None of the conclusions drawn in the study is affected by these changes.

To examine the sensitivity of convection to ambient relative humidity, we also conducted a sequence of lowresolution $2 \mathrm{D}$ runs with the relative humidity above $2 \mathrm{~km}$ reset to a vertically uniform value between $25 \%$ and $90 \%$. The changes shown by these simulations will be compared to those of an idealized parcel calculation in section 4 .

\section{b. Updrafts}

The peak vertical velocity $w$ versus time $t$ and height $z$ is shown in Figs. 1 and 2 for the 2D low-resolution case (2DLR) and the 3D high-resolution case (3DHR). As we are not interested in documenting the resolution and 


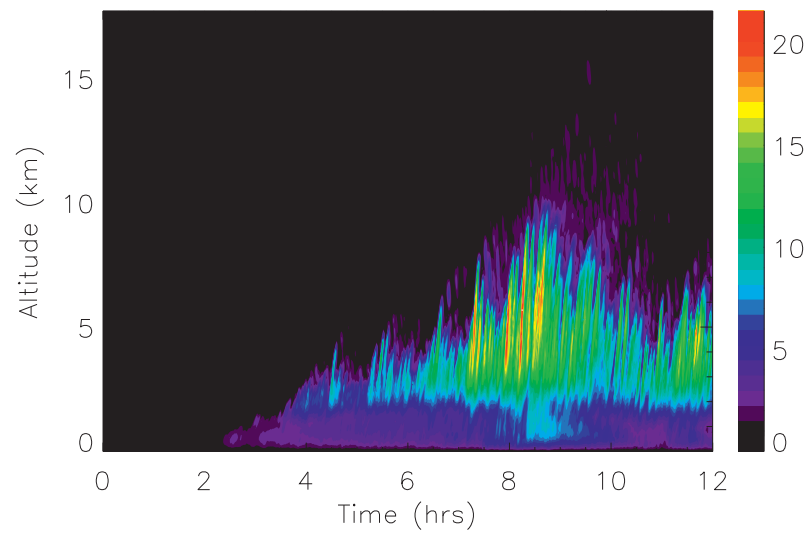

FIG. 2. As in Fig. 1, but for the 3DHR simulation.

dimension dependence of the results per se we do not show the other runs; their behavior is generally intermediate between the two shown here.

In all runs the convection begins and gradually deepens over a period of a few hours, eventually reaching the upper troposphere, though not all the way to the tropopause. The deepening always occurs in a sequence of multiple thermals each reaching higher than the last, visible as slanted narrow streaks in the figures. Convection reaches several kilometers higher in the $2 \mathrm{DLR}$ than in 3DHR, and the drafts are broader in the 2DLR case. In 2DLR, several strong drafts reach near 10 $15 \mathrm{~m} \mathrm{~s}^{-1}$; in 3DHR most drafts show a similar peak speed, but there are a few that get up to $20 \mathrm{~m} \mathrm{~s}^{-1}$ or so. Differences are also apparent in the boundary layer, which is not as well resolved in the coarse runs.

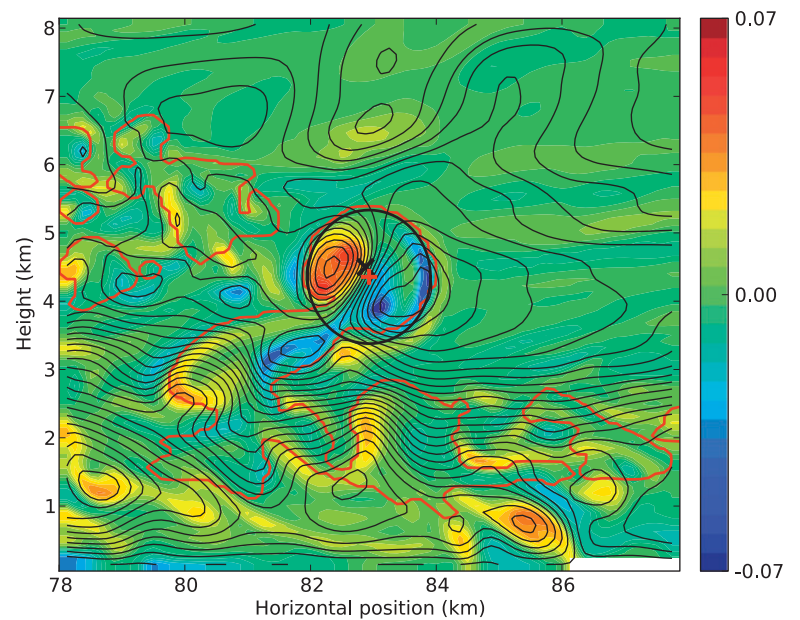

The updraft characteristics shown in these figures will be revisited later when we discuss the predictions of parcel models in section 4. For now we note that updrafts remain narrow throughout their ascent and usually reach their peak velocities near 5-6 km.

\section{c. Thermal structure}

The individual thermals seen in the vertical velocity plots closely resemble those described by previous researchers (Scorer and Ronne 1956; Blyth et al. 2005; Damiani et al. 2006). An example is indicated in Fig. 3, which shows a compact, quasi-circular vorticity dipole and similarly compact cloud water field. In particular this resembles the classical Hill's vortex (Hill 1894) -an exact solution of the Navier-Stokes equations in which a spherical bubble slips through the surrounding fluid by executing a toroidal, internal circulation like that of a very thick smoke ring (see Batchelor 1967). This structure implies an upward velocity peaking on the vertical center axis-where it is at least double the rise rate of the thermal-and decreasing to small negative values at the thermal's equator (side of the cloud). These predictions are confirmed by cloud observations (Turner 1973, 186-196; Keenan and Carbone 1992).

\section{d. Estimation of the thermal's boundary}

It is necessary to set the thermal's boundary in order to compute most of the quantities that we are interested in. As discussed in section 1, a problem with conditional sampling methods typically used is that the active regions so identified may differ significantly from those of

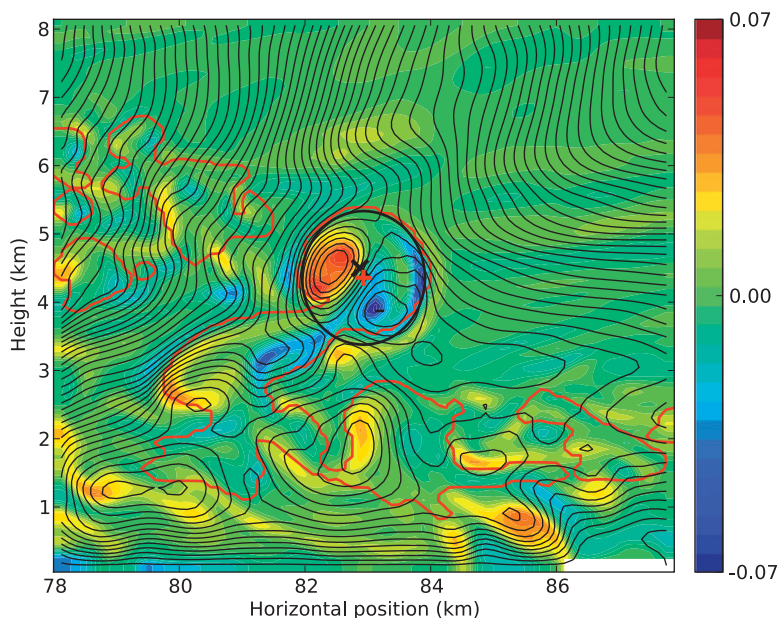

FIG. 3. A snapshot of thermals at 463 min into the 2DHR simulation. Coloring indicates vorticity $\left(\mathrm{s}^{-1}\right)$. Black contours show streamlines in a reference frame that is (a) fixed and (b) moving with the main thermal. The black " $\times$ " denotes the point of maximum updraft velocity. The red plus sign denotes the estimate of the center of the thermal, and the circle is its estimated boundary according to the method described in section $2 \mathrm{~d}$. Fluid velocity is tangent to streamlines and inversely proportional to streamline spacing. Red contours show clouds (regions where cloud water content $>0.1 \mathrm{~g} \mathrm{~m}^{-3}$ ). 

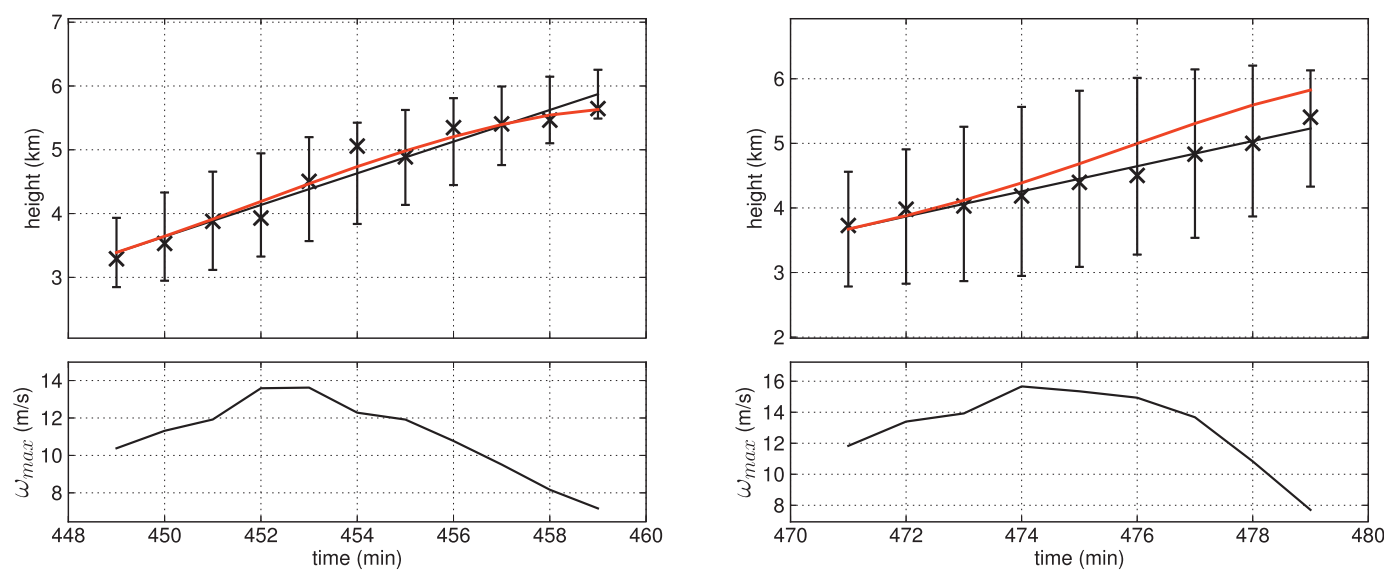

FIG. 4. Two examples of our estimate of the height of the thermal's center and its radius taken from the 3DHR simulations. (top) Crosses indicate the position of the strongest updraft. The linear fit (black line) of slope $W$ is used as the estimated center, and the error bars show the vertical extent of the thermal about the fitted position. The red line indicates the expected trajectory according to the momentum budget. (bottom) The evolution of the peak vertical velocity $w$.

the actual rising air mass as described above. In particular, there are likely to be vigorous exchanges of mass and momentum between the rising (active) and falling (partly inactive) portions of a thermal that are irrelevant to the trajectory of the thermal as a whole. Therefore, rather than attempt to define an exact cloud boundary, we aim to draw an approximate boundary of the vortex ring or dipole.

In light of the nearly spherical or circular appearance of the thermals, we choose circles (2D) or spheres (3D) as bounding surfaces. To estimate the position of the center of the thermal at time $t$, we first trace the location $(x, y, z)$ of the strongest $w$ at each time during which the thermal is clearly identifiable. These points are found a posteriori to rise at an approximately constant rate during this part of the thermal lifetime (see Fig. 4). A linear approximation $z_{\text {therm }}=W t+b$ therefore provides a reasonable estimate of the height of the thermal's center, with the slope $W$ giving the thermal's (constant) ascent rate.

We then determine the thermal's radius $R(t)$ by finding, at each time $t$, the $R$ for which the average of $w$ inside the enclosed volume matches the ascent rate $W$. Visual inspection of animated plots of the estimated circles superimposed on the vorticity and cloud water fields suggest that this method correctly tracks the position and spatial extent of the thermals (see Fig. 3 for an example of a snapshot of this; a full movie is available as supplemental material at the Journals Online website: http://dx.doi.org/10.1175/JAS-D-12-0220.s1).

We applied this method during the cumulus growth phase (i.e., around $6-8 \mathrm{~h}$ into the simulation) in which we could identify clearly defined thermals that would survive for more than 5-6 min. In total, we tracked seven thermals in the 2DHR simulation and eight thermals in the 3DHR simulation, for a total of $68 \mathrm{~min}$ of data from the 2DHR simulation and $72 \mathrm{~min}$ from the 3DHR simulation. Typical initial tracking heights were between 2 and $3 \mathrm{~km}$, followed up by roughly constant rising rates of about $4-5 \mathrm{~m} \mathrm{~s}^{-1}$. Thermals survived usually around $8-10 \mathrm{~min}$ before breakup, reaching heights of $4.5-5.5 \mathrm{~km}$ in most cases. This tracking period does not fully cover the apparent thermal lifetimes; we are still working on additional strategies to track the thermals over longer times. In particular, thermals were seen in the upper troposphere later as the congestus deepened, as suggested in Figs. 1 and 2. Such thermals, however, were difficult to track consistently from low levels (see section 3c).

Peak vertical velocities took place close to the middle of the tracking period, roughly at heights between 3.5 and $4.5 \mathrm{~km}$. Although we only tracked a limited number of clearly identifiable thermals, visual inspection of the time evolution of the simulated convective regions suggests that thermal-like flow features eventually occur in the upper troposphere and dominate the overall convective activity at lower levels. This can be seen in an animation of a fragment of the 2DHR simulation showing the vorticity and cloud water field (available at the Journals Online website: http://dx.doi.org/10.1175/JAS-D-12-0220.s2).

\section{e. Mass entrainment}

\section{1) THermal GROWTH}

While convective schemes incorporate a variety of entrainment and detrainment assumptions, it is typical 
in deep convective schemes for net entrainment to occur during plume ascent. This leads to a growth of mass flux with height. Such schemes often employ a bulk plume meant to represent the net effect of an ensemble of clouds; as such, the bulk entrainment or other process need not behave like that of any single cloud. Nonetheless, such schemes must ultimately be founded to some extent on assumptions as to the behavior of individual clouds.

The individual thermals we tracked in the CRM show little net mass growth with height, rather maintaining a characteristic scale of about $2 \mathrm{~km}$. This can be seen in Figs. 1 and 2; although some updrafts do grow with height they generally remain thin. Interestingly, the $2-\mathrm{km}$ scale roughly matches the two most obvious scales imposed by the atmosphere: the boundary layer thickness and the scale height of water vapor. We doubt this is a coincidence. The presence of such external scales is ignored in traditional plume analogs, where the only relevant length scale is the distance from the mass source (nozzle). In such cases the plume diameter would be expected to scale with altitude, contrary to the behavior calculated in the CRM. A complicating factor in assessing parameterizations is the issue of how bulk properties may differ from those of individual thermals, discussed further in section 5 .

The failure of thermals to grow as expected from laboratory studies of individual dry thermals (Sánchez et al. 1989; Turner 1957) begs the question of whether those found here are entraining at the high rates reported elsewhere, and how latent heat release might be altering the dynamics. We now turn to these questions.

\section{2) ENTRAINMENT DisTANCES/RATES}

We estimated thermal entrainment rates (as well as several other quantities described subsequently) using the thermal boundaries described in section $2 \mathrm{~d}$ - that is, circles (in 2D) or spheres (in 3D) with time-dependent radii. At each time step we calculated the rate at which mass was being exchanged through the region boundaries, and divided this by the mass within the region to obtain an entrainment rate (per unit time). The average upward velocity within the region, divided by this entrainment rate, yields an entrainment distance $D_{\text {entrain }}$. Over this distance, the proportion of original fluid inside the region would be reduced by a factor of $e$ (if the fluid leaving the region were representative or random) or to zero (if the oldest fluid left first).

To calculate entrainment we use the air velocity $\mathbf{u}^{\prime}$ in the frame of reference that is moving with the tracked thermal (i.e., $w=W+w^{\prime}$ and similar for the other two components of $\mathbf{u})$ :

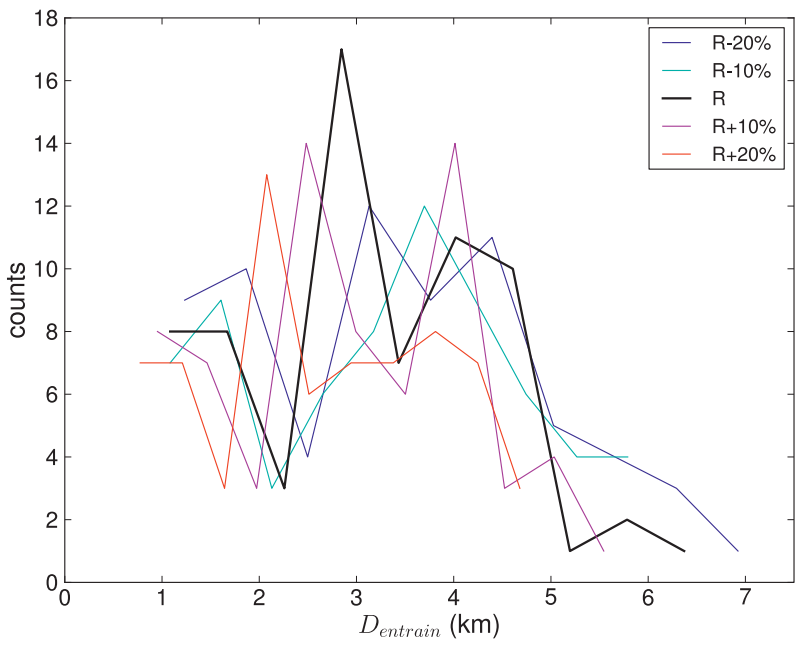

FIG. 5. Histogram of entrainment distances measured on the tracked thermals of the 2DHR simulation. Thick black line corresponds to the radii as estimated by our method, while the different colors correspond to the modified radii, as described in the legend.

$$
D_{\text {entrain }}=\frac{\int_{V} \rho w d V}{\oint \mathbf{u}^{\prime} \cdot \hat{\mathbf{n}} \times H\left(\mathbf{u}^{\prime} \cdot \hat{\mathbf{n}}\right) \rho d S},
$$

where the contour and volume integrals are evaluated on the boundary $S$ and interior $V$ respectively of the circular (for 2D) or spherical (for 3D) control region, and $H(\cdot)$ is the step function (only air flowing into the volume is counted). The density $\rho$ accounts for the different molecular weight of water vapor and includes the mass of condensed water (which is more important for the calculations in section 3 ).

This estimate must be regarded as crude; the distance could be too short due to unwanted contributions from transient distortions of the thermal shape, or too long if there is additional entrainment occurring due to unresolved eddies. We know of no significantly better way to estimate entrainment without inserting artificial tracers specifically for this purpose as done by Romps and Kuang (2010), or defining the cloud boundaries through active and inactive regions (Romps 2010; Dawe and Austin 2011), which, as discussed earlier, we wish to avoid.

We obtained entrainment distances of $1-5 \mathrm{~km}$ in the 2DHR simulation, and of $1-4 \mathrm{~km}$ in the 3DHR simulation, with most frequent values falling around $3 \mathrm{~km} \mathrm{(2D)}$ and $2 \mathrm{~km}$ (3D) (Figs. 5 and 6). Note that the distribution of the calculated entrainment distances in the 2DHR simulation shifts slightly as we modify the estimated radius of the thermal: assuming smaller circles results in longer entrainment distances (lower rates), and larger circles result in shorter distances (higher rates). 


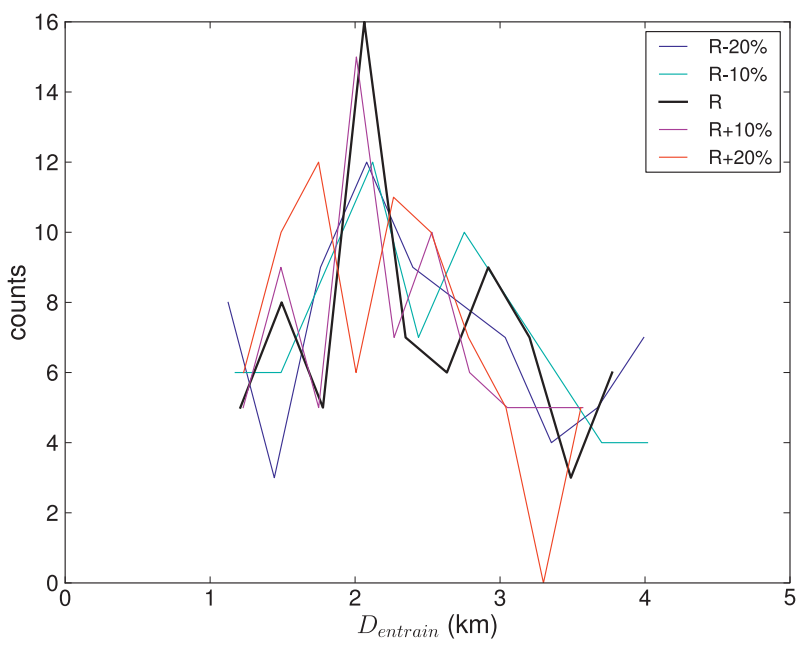

FIG. 6. As in Fig. 5, but for the 3DHR simulation.

This size dependence is weak, however, and almost disappears in the 3DHR simulation.

These rates are quantitatively similar to those obtained by previous researchers using different analysis methods and models, and were similar across the resolutions examined, as found by previous studies looking at this more carefully (Romps and Kuang 2010). They are also supported by results shown in section 4 . Rates were insensitive to the control region radius, suggesting a robust measure. Runs with a range of initial humidities aloft showed that the entrainment rate was not very sensitive to this. Further work will examine entrainment rates more carefully, but for the present study it is sufficient to confirm that they are consistent with those of previous studies. In section 4 we will present results that indirectly support these entrainment rates.

\section{Vertical momentum budget}

We first present a heuristic discussion of various drag forces and then proceed to results.

\section{a. Should thermals experience form drag?}

One aspect of a rising blob that distinguishes it from a slender plume is the expectation, frequently found in the literature, that the former will experience form drag. However, analyses of clouds in numerical simulations often do not find large nonhydrostatic drag forces (e.g., $\mathrm{Xu}$ and Randall 2001).

A crucial feature of Hill's vortex is that the internal and external flows match everywhere along the spherical stream surfaces defining the material boundary of the thermal, such that the velocity is continuous across the interface between vortex and the environment. This is crucially important because it eliminates the viscous boundary layer that would occur adjacent to a solid body (see Batchelor 1967).

At high Reynolds numbers, it is this boundary layer that gives rise to the form drag experienced by aircraft, golf balls, and other solid objects. The drag arises from power dissipated in continually generating finescale turbulence in the boundary layer, which forms a steadily growing wake behind the object (Batchelor 1967). The deformation of flow around the object outside of this boundary layer (approximated by a potential-flow solution to the NavierStokes equations and unaffected by the internal circulation of the thermal) does not generate sufficient shear to be a significant additional source of drag at the high Reynolds numbers of interest here.

Since the shear layer causing drag does not form around a Hill's vortex possessing an internal matching circulation, there is no reason to expect significant form drag on a compact vortex at high Reynolds number (Turner 1964). Indeed, modern laboratory studies demonstrate this unequivocally: for example, Dabiri and Gharib (2004) found that laboratory thermals closely approximating perfect Hill vortices could propagate a distance equal to many times their diameter with little if any form drag. This phenomenon will also be familiar to anyone who has watched the propagation of a smoke ring, which decelerates only very modestly while traveling many diameters.

\section{b. Should thermals lose momentum through mixing?}

If a cloud entrains motionless ambient fluid, its upward momentum will be diluted by the added mass, decreasing its upward velocity in the absence of body forces (Turner 1972). Some drag of this type is incorporated into every formulation of a cloud model known to the authors that includes an explicit vertical momentum equation. This dilution of momentum is not severe for low entrainment rates but would be stifling at the entrainment rates of $(2 \mathrm{~km})^{-1}$ found here and in previous studies.

However, our thermals did not grow as rapidly as would be implied by entrainment alone (section 2e), which complicates the situation since it implies significant detrainment. Nonetheless, under traditional assumptions an exchange of material between the thermal and environment should export upward momentum from the parcel and produce a downward acceleration $\left(F_{\text {res }}<0\right.$, where $F_{\text {res }}$ denotes the resolved convergence of vertical momentum flux).

A detraining thermal, however, with its peculiar circulation, is capable (at least in principle) of something very counterintuitive: it can mix strongly with its environment in terms of material properties but keep $F_{\text {res }}$ close to zero. Upward moving air is buried within the core of the thermal, not at its edge where it can mix with 

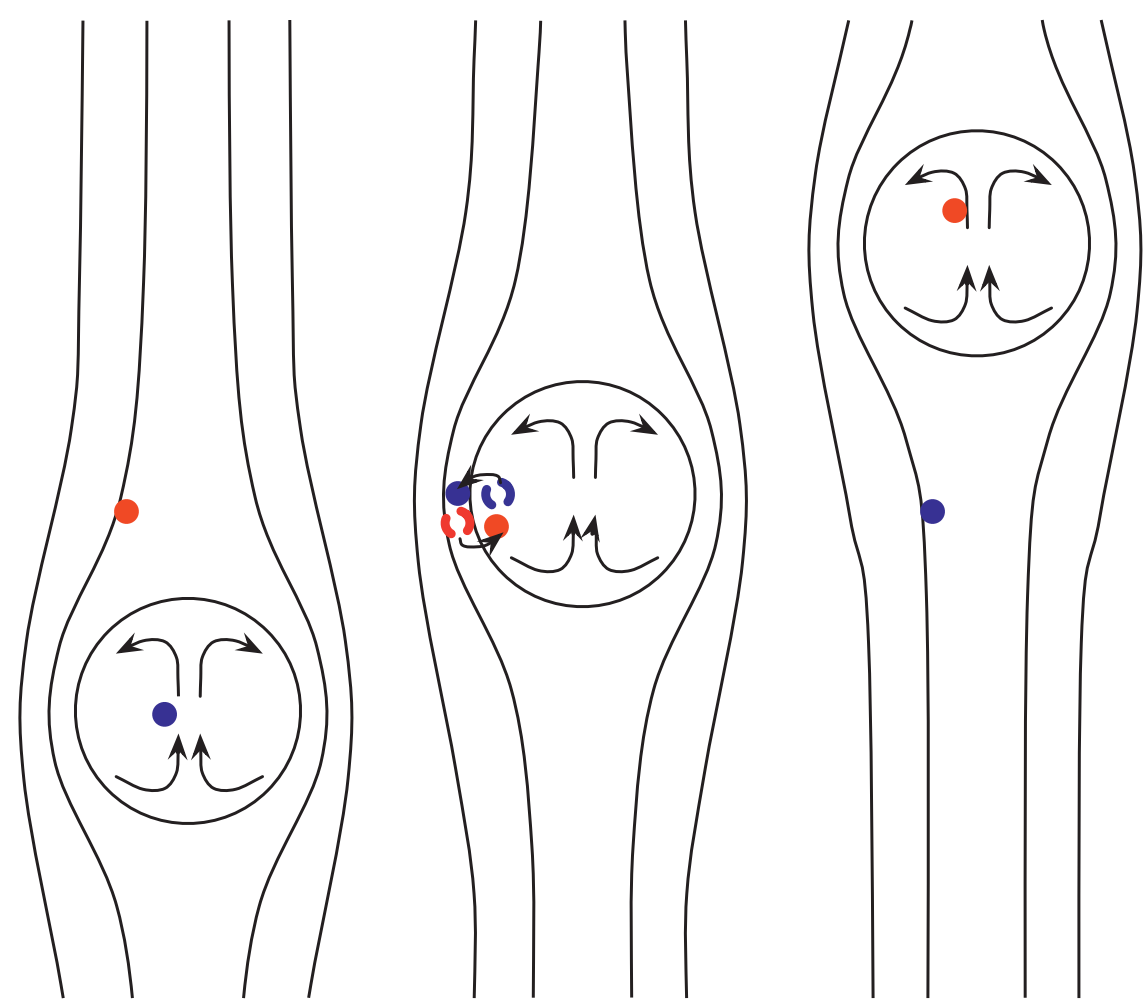

FIG. 7. Conceptual illustration of how a thermal vortex can exchange mass with the surrounding airstream without losing momentum. (left to right) Pictures before, during, and after exchange. Blue and red dots mark air parcels originally inside and outside the thermal, respectively. Mass exchange is shown near the equator of the thermal but could occur anywhere along its boundary, affecting the provenance of air subsequently drawn into the main updraft as found in laboratory studies.

the environment. By the time air approaches the sides of the thermal, where exchanges with surrounding air are typically observed to occur in the laboratory (Turner 1973, 186-196) and in simulations (Fig. 3; see also Heus et al. 2008), the air has transferred all of its momentum to other air still in the thermal. The mass exchange would thus be between two air masses neither of which has significant upward momentum, producing no deceleration of the thermal (see Fig. 7).

Note that strong shears do exist in the thermal vortices (evident from the vorticity distributions in Fig. 3), but contrary to the common picture in which the shear lies primarily at the edges of the cloud, in a Hill vortex-type thermal the shear is well within the interior of the thermal. Shear there should dissipate the thermal's angular momentum or vorticity, but without directly affecting its average upward momentum. Angular momentum, however, is constantly being regenerated by the concentration of latent heat release in the central, upwardmoving portion of the thermal. In summary, it seems plausible that cumulus parcels could avoid some or all of the naively expected momentum dilution if they resemble Hill-type vortices.

\section{c. Budget calculation}

In light of the above questions surrounding drag on air parcels, we examine the vertical momentum budget for each tracked thermal. The vertical velocity equation for the control regions discussed in section $2 \mathrm{~d}$ can be written as

$$
\frac{d W}{d t}=\frac{1}{M} \oint p n_{z} d S-g+F_{\text {res }}+F_{\text {unr }}
$$

where $M=\int_{V} \rho d V$ is the total mass within the control region, and $n_{z}$ is the vertical component of the normal vector perpendicular to the surface $d S$. The first term on the right-hand side is the vertical component of the pressure gradient force per unit mass acting on the thermal's volume, which together with $-g$ comprises the buoyancy plus any nonhydrostatic accelerations such as form and wave drag. The term $F_{\text {res }}$ denotes the convergence of vertical momentum flux resolved by the model, and $F_{\text {unr }}$ that of the unresolved fluxes, which we assume negligible. In particular, we are interested in $F_{\text {res }}$, which measures the momentum impact on the rising thermal due to mixing. We compute it directly as 


$$
F_{\text {res }}=\frac{\oint \mathbf{u}^{\prime} \cdot \hat{\mathbf{n}} \rho w^{\prime} d S}{M} .
$$

Additionally, we can estimate a buoyancy acceleration on the control volume:

$$
B=-\frac{1}{M} g \int_{V}\left(\frac{\rho-\bar{\rho}}{\bar{\rho}}\right) \rho d V
$$

where $\bar{\rho}$ is the average density outside the thermal at the same altitude. Note that buoyancy is never unambiguously defined since the choice of environment (here, the entire domain outside the thermal) is arbitrary. By subtracting this buoyancy from the first two terms in Eq. (2), we obtain the residual nonhydrostatic components of the pressure gradient force per unit mass, $F_{\text {nh }}=(1 / M) \oint p n_{y} d S-g-B$, which constitute another possible source of drag in addition to $F_{\text {res. }}$. These nonhydrostatic forces would include form drag (which should be negligible on physical grounds for a Hill-type vortex) and wave drag (which results from environmental stratification, not usually present in laboratory experiments examining such vortices). An alternative form of Eq. (2), neglecting unresolved fluxes, is thus

$$
\frac{d W}{d t} \approx B+F_{\mathrm{nh}}+F_{\mathrm{res}} .
$$

Figure 8 shows an example of how the different terms in Eq. (5) evolve throughout the lifetime of a thermal in the 3DHR simulation. The flux convergence term $F_{\text {res }}$ is small and positive; that is, it accelerates the thermal slightly, in direct contrast to the traditional assumption. The main source of drag is $F_{\text {nh }}$, as also found recently by de Roode et al. (2012).

Figures 9 and 10 show histogram plots for the three terms on the right-hand side of Eq. (5) and their sum, obtained from the 2DHR and 3DHR simulations, respectively. In all simulations examined, regardless of the control volume size, $F_{\text {res }}$ usually accelerated the thermal. Such accelerations were weak, usually below $\sim 0.01 \mathrm{~m} \mathrm{~s}^{-2}$ (equivalent to $\sim 0.3 \mathrm{~K}$ of buoyancy). This unexpected result has been noted previously in simulated shallow convection (Schumann and Moeng 1991; de Roode et al. 2012), where it was attributed to macroscale motions (resolved by the calculations) but was not explained. This indicates that the absence of the expected entrainment drag is not an artifact of our tracking methodology. Results were qualitatively similar for all simulations done here. Circle sizes do not affect much the distribution of $F_{\text {res }}$, in particular its mean. The same holds for the distributions of the other terms in the 2DHR simulation; on the other hand,

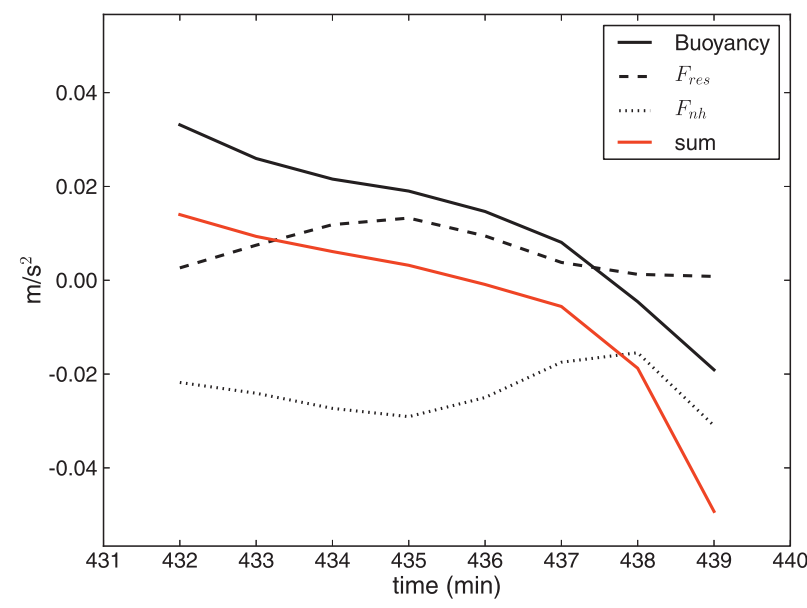

FIG. 8. Example of the time evolution of the vertical momentum budget terms in Eq. (5) for a thermal in the 3DHR simulation.

buoyancy and $F_{\mathrm{nh}}$ are affected by the chosen radii in the 3DHR simulation. However, note that this dependence is opposite in these two terms, such that the total sum remains unchanged.

The net upward force histogram peaks around zero, consistent with the fact that we traced thermals as they rose with approximately constant vertical velocities. This suggests that we are indeed capturing the relevant contributions to the vertical momentum budget, and that neglecting the unresolved fluxes is a valid approximation. To illustrate this further, red lines in Fig. 4 depict the thermal's expected ascent according to the vertical momentum equation, given its initial $W$. Our finding of small convergence of vertical momentum fluxes is supported by studies showing that pressure gradient forces dominate over shear forces in the vertical momentum budget (Schumann and Moeng 1991).

Buoyancy accelerations in our simulations typically varied between -0.01 and $0.02 \mathrm{~m} \mathrm{~s}^{-2}$ with a mean near $0.01 \mathrm{~m} \mathrm{~s}^{-2}$. This was counteracted primarily by the nonhydrostatic force, leading to small net accelerations consistent with the approximately constant rise rates observed over the tracking periods.

Dividing the typical $W$ of $\sim 4 \mathrm{~m} \mathrm{~s}^{-1}$ by the mean buoyancy yields an acceleration time scale of $\sim 400 \mathrm{~s}$, which is not significantly less than the typical thermal lifetime. Thus, the thermal motion is only weakly damped even if buoyancy is considered as its sole driver. The true driving force for the thermal may however be significantly less than this because the nonhydrostatic resistance does not necessarily act as a passive drag. While part of it represents an irreversible loss of energy via radiated waves or turbulence, part will appear as inertia in nearby motions (e.g., "compensating subsidence") that could be returned to the parcel later when it decelerates. Further work will be 

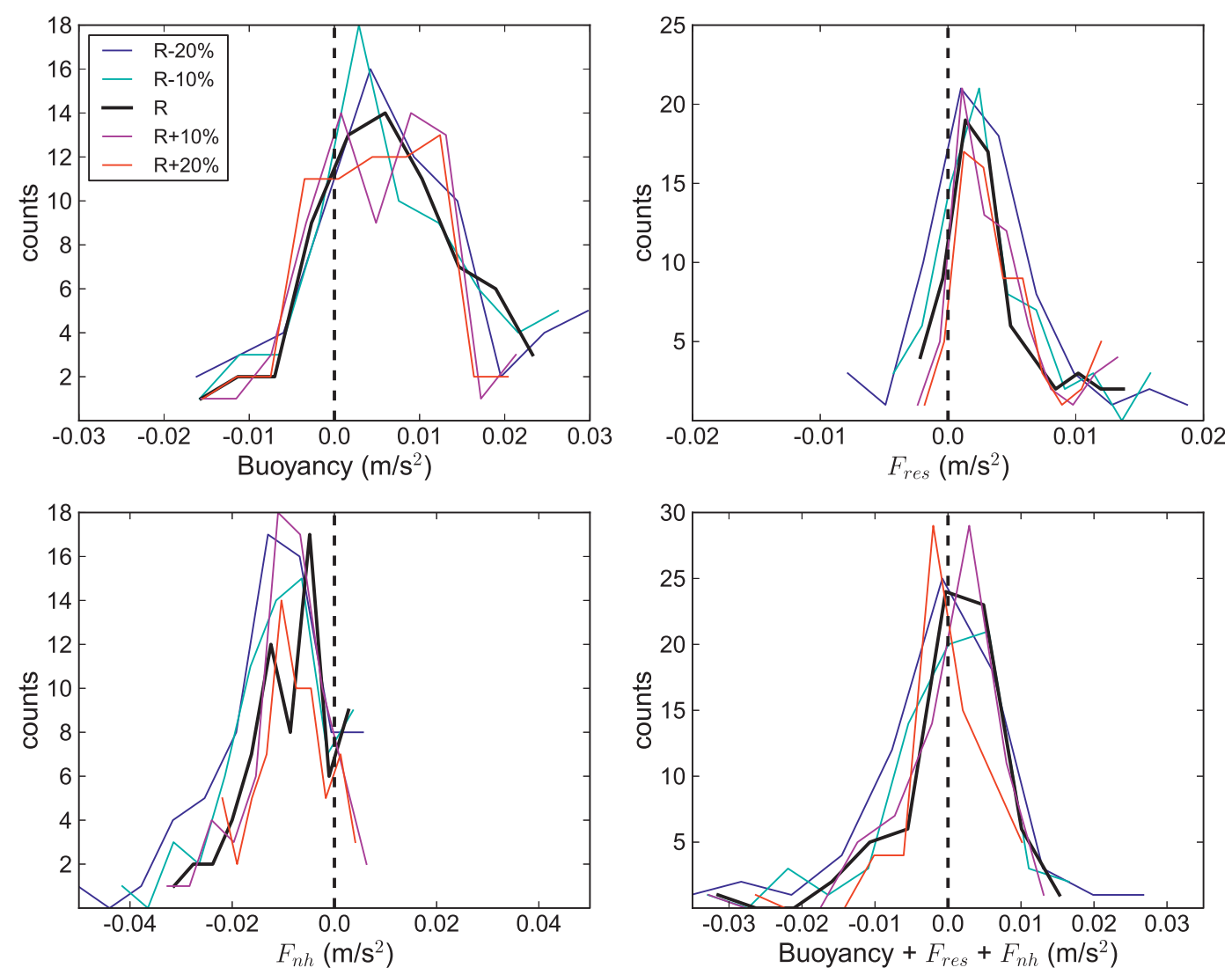

FIG. 9. Histograms of the different terms in the vertical momentum budget [Eq. (5)] for the tracked thermals in the 2DHR simulation: (top) (left) buoyancy and (right) $F_{\text {res }}$; (bottom) (left) $F_{\text {non-h }}$ and (right) buoyancy $+F_{\text {res }}+F_{\text {non-h. }}$. The thick black line corresponds to the values obtained when using the radii as estimated by our parcel method, and the different colors correspond to modified radii, as indicated by the legend. The vertical dashed line indicates the zero crossing.

necessary to quantify these two alternatives and determine how "slippery" the thermals really are.

One may also ask how typical these tracked thermals are of structures that produce upward convective mass flux generally. We repeated our calculation of the momentum flux convergence for the regions surrounding the strongest $w$ in the domain at each time step throughout every simulation and found similar results throughout, including during deep convection. Thus we find the absence of mixing drag to be a general characteristic of convective updrafts.

Although the thermals tracked here only reached about $7 \mathrm{~km}$, later in the simulation, thermals having similar characteristics reached as high as $10 \mathrm{~km}$. However, we were not able to track any single thermal from the boundary layer to the upper troposphere despite the coherency of the ascending patterns in Figs. 1 and 2. Moreover, while $W$ remains fairly constant over the tracking intervals, $w$ is strongest in the middle of the interval (Fig. 4). This suggests the thermals undergo episodic transitions of some kind, which would be worth further investigation. It also shows that the dynamics of the entire thermal parcel appear simpler than those governing the core updrafts that have been the focus of previous studies.

\section{Parcel-model comparison: Standard case versus detraining thermal vortex}

Now we explore the implications of different amounts of drag for deep convective behavior, by calculating the fate of a simple pseudoadiabatically ascending parcel beginning at $980 \mathrm{hPa}$ with a specified initial ascent rate, with and without entrainment drag. The parcel ascends through a mean observed tropical sounding with modest CAPE (the same one used to initialize the numerical simulations), except that the sounding is modified by resetting the relative humidity above $2 \mathrm{~km}$ to a constant value ranging from $60 \%$ to $100 \%$, to test the sensitivity of the result to ambient relative humidity.

Our basic assumptions are standard except for two. First, we adopt an effective mass for the parcel 1.5 

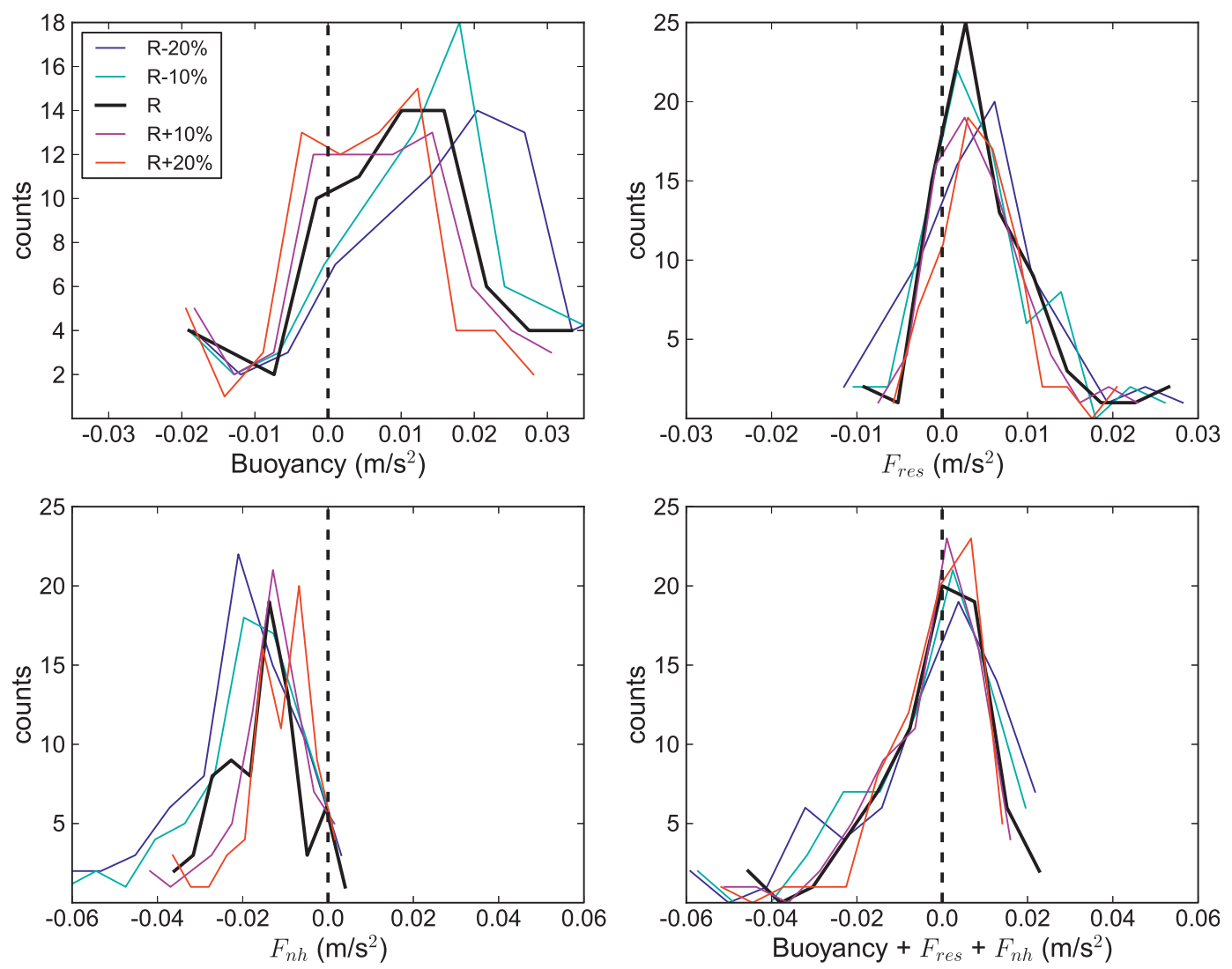

FIG. 10. As in Fig. 9, but for the 3DHR simulation.

times its actual mass, to account for that of surrounding air that must move out of the way (Batchelor 1967; Romps and Kuang 2010). (Note that these environmental motions are often incorrectly invoked as a source of drag, but their principal effect is only to add inertia.) Second, we retain the internal heterogeneity of the thermal rather than mixing it. This has little effect on results but enables us to compute the fraction of the thermal that is cloudy (conventionally, this fraction is implicitly unity). Entrainment rates are assumed vertically uniform, and form and wave drag are neglected for simplicity.

\section{a. Parcel material properties}

\section{1) Homogeneous PARCEL}

The usual assumption in parcel calculations is that the parcel is well mixed and is represented by a single composition and temperature. The moist entropy $\theta_{e}$ of a uniform air parcel, a function of height $z$, is conserved upon ascent and is approximately conserved upon mixing with the environment such that (e.g., Sahany et al. 2012)

$$
\frac{d \theta_{e}}{d z} \approx-\epsilon\left(\theta_{e}-\overline{\theta_{e}}\right),
$$

where $\epsilon$ is the entrainment rate (reciprocal of entrainment distance) and overbars denote environmental values. Having determined $\theta_{e}$ from this equation, the temperature $T$ above cloud base is found that yields this $\theta_{e}$ with saturation water vapor mixing ratio $r_{s}(T)$; that below cloud base is found be taking $r$ to be the same as its initial value. The air parcel's buoyancy $B$ is then approximated as

$$
B=g\left(\frac{T_{v}-\overline{T_{v}}}{\overline{T_{v}}}\right),
$$

where $T_{v}$ is the virtual temperature. For simplicity, the buoyancy effects of condensed water due to its heat capacity, latent heat of freezing, and gravitational loading are all neglected here. If included, they would tend to reduce buoyancy at low levels and increase it at higher levels, but their calculation introduces problems since not all condensed water remains in the parcel, especially at low entrainment rates where condensed water amounts are unrealistically large (see below).

\section{2) INHOMOGENEOUS PARCEL}

While the above calculation is made here for consistency with existing work, we prefer an alternative where 
the air parcel is not assumed to undergo any internal mixing. In this case the parcel remains a heterogeneous assemblage of all the different air types it has entrained during its history. To implement this we calculated nonentraining parcel quantities for all starting levels, to obtain a matrix of values for every initial and final level in the absence of mixing. The condensed water content of each component is just its adiabatic water content.

We then calculate the proportion per unit distance (mixing fraction) $P_{z}$ of the air at a final level $z$ that originated at each starting level $z^{\prime}$ as

$$
P_{z}\left(z, z^{\prime}\right)=\epsilon \exp \left[\epsilon\left(z^{\prime}-z\right)\right],
$$

and the proportion originating near the surface as

$$
P_{0}(z)=\exp \left[\epsilon\left(z_{0}-z\right)\right] .
$$

The mean properties of the parcel at level $z$ are then calculated as the weighted average over $z^{\prime}$ of the individual component properties, with weighting function $P_{z}$; for example,

$B(z)=P_{0}(z) B\left(z, z_{0}\right)+\int_{z_{0}}^{z} B\left(z, z^{\prime}\right) P_{z}\left(z, z^{\prime}\right) d z^{\prime}$.

Comparisons with the homogeneous calculation (not shown) indicate that the buoyancies and ascent rates for air parcels are little affected by the traditional assumption of homogeneity. However, the heterogeneous calculation enables a prediction of cloud amount: the fraction of air inside the thermal that has reached its lifting condensation level. The degree of internal mixing is also crucial for cloud microphysics (e.g., Paluch and Baumgardner 1989) although that is not considered here.

\section{b. Parcel momentum}

Once buoyancies are obtained by either of the above methods, conservation of momentum gives

$$
\frac{d W}{d z}=\frac{1}{W} \frac{d W}{d t}=\frac{B}{W}-\epsilon_{w} W,
$$

where $\epsilon_{w}$ is the drag coefficient. The standard assumption is to set $\epsilon_{w}=\epsilon$, or a Prandtl number $\operatorname{Pr}=1$, since momentum and thermal properties are assumed to be exchanged by the same air currents, and these are conventionally assumed to carry the average properties of their region of origin (cloud or environment). We retain here the usual assumption that the environment is at rest.

Alternatively, we consider the extreme case of $\epsilon_{w}=0$ but with the same dilution of buoyancy (section 4a). This is equivalent to setting the turbulent Prandtl number to zero.
Note that most convection schemes do not even use an explicit momentum equation of the form given above for $W$. Shallow convective parameterizations sometimes use an alternative equation for $W$ with two terms whose coefficients are empirically tuned (see, e.g., de Roode et al. 2012). Most current deep-convective schemes do not calculate $W$ explicitly, often simply assuming that updrafts stop at their neutral buoyancy level, which is equivalent to assuming a very strong mechanical drag or damping. Thus even our "standard" calculation allows a greater role for inertia than would be found in many schemes.

\section{c. Results for three illustrative parcel models}

We perform parcel calculations for three cases:

- no entrainment;

- a standard model, in which the Prandtl number is unity (the usual assumption) and entrainment is weak $\left[(10 \mathrm{~km})^{-1}\right]$; and

- a new model, in which the Prandtl number is zero and entrainment is strong $\left[(2 \mathrm{~km})^{-1}\right]$.

Cloud models used in deep convective parameterization typically assume there are drafts with a range of entrainment rates, usually including zero. Since the nonentraining draft reaches the highest, its neutral level is often taken to establish the thickness of the layer affected by convection. The low entrainment rate chosen here for the standard model yields overall undilute transport rates roughly characteristic of some current convective schemes (Romps and Kuang 2010) although individual schemes will differ. The strong entrainment rate in the new model roughly matches those found in numerical simulations. We find that this high entrainment does not permit deep convection with the standard momentum loss assumption, as the parcels lose buoyancy and momentum too rapidly. Figure 11 compares the results of these calculations for an initial parcel velocity of $7 \mathrm{~m} \mathrm{~s}^{-1}$.

Note that we do not explicitly consider mass fluxes here, but simply calculate thermal properties at each height. Exponential mass growth of the thermal is implicit in the traditional assumption, but not in the new model.

\section{1) Cloud height And vertical Velocity}

The nonentraining parcel, used by many current schemes to establish the greatest height reached by convection, is neutrally buoyant near $15 \mathrm{~km}$ regardless of ambient humidity. Our "standard" model with low entrainment also predicts clouds reaching deeply; the height is modestly affected by ambient humidity, but clouds still reach the upper troposphere even when relative humidity is only $60 \%$. 


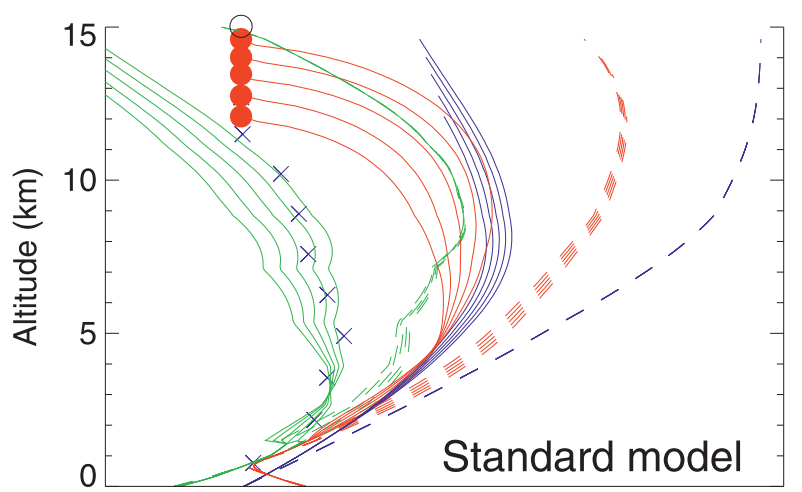

\begin{tabular}{|c|c|c|c|c|c|}
\hline-0.1 & 0 & & & & \\
\hline \multirow[t]{2}{*}{10} & 0 & 10 & 20 & 30 & 40 \\
\hline & 0 & 5 & 10 & 15 & 20 \\
\hline
\end{tabular}

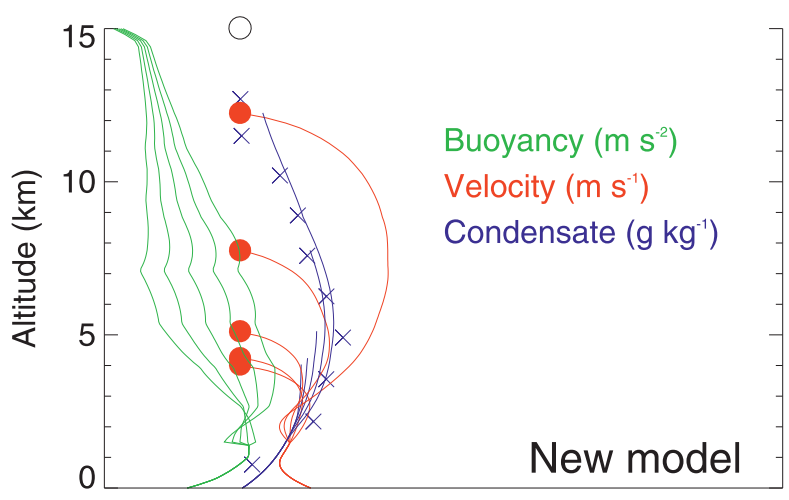

FIG. 11. Calculations for an ascending air parcel. (top) The standard model with entrainment rate $\epsilon=(10 \mathrm{~km})^{-1}$ (solid) or 0 (dashed). (bottom) The new model with $\epsilon=(2 \mathrm{~km})^{-1}$. Green, red, and blue curves show respectively the buoyancy, upward velocity, and condensed water content of the parcel/thermal, for five values of surrounding relative humidity above $2 \mathrm{~km}$ (from lowest to highest: $60 \%, 70 \%, 80 \%, 90 \%$, and $100 \%$ ). Blue crosses show maximum total cloud water content in the explicit cloud simulations. The black open circle shows the zero-buoyancy level of a nonentraining parcel; filled dots show the maximum height reached by the parcel including overshoot. Initial parcel velocity is $7 \mathrm{~m} \mathrm{~s}^{-1}$ in all cases.

The new model, by contrast, predicts that clouds transit the upper troposphere only in very humid environments, otherwise stopping in the middle or lower troposphere. This sensitivity to ambient humidity aloft is shown in observations (e.g., Holloway and Neelin 2009; Sherwood et al. 2010), and by CRMs, while the standard results are characteristic of climate model representations (Derbyshire et al. 2004). Note that the final cloud outflow would mostly occur below the overshoot height shown, although possibly not far below if sufficient mixing occurred during the overshoot. The new-parcel ascent profile in the most humid environment resembles that of the standard parcel in the driest environment.
Note also that by the time convection becomes deep, earlier convection has had time to raise the local humidity at midlevels so later thermals will encounter a moister environment than the initial one. It is thus reasonable that a high humidity is needed for parcels to reach the upper troposphere.

Above a few kilometers, standard parcels continue to accelerate until reaching peak velocities of $15-20 \mathrm{~m} \mathrm{~s}^{-1}$ at levels from 5 to $10 \mathrm{~km}$. New-model ascent profiles are more sensitive to humidity, and generally slower, not attaining $10 \mathrm{~m} \mathrm{~s}^{-1}$ except in the most humid environment. They are closer to those found in the CRM than are the standard ones. In both calculations (especially the standard one), the parcel decelerates quickly in the first kilometer as a result of convective inhibition, and then accelerates. This early behavior is sensitive to details of the sounding, which are no longer the same by the time convection deepens as they were in the initial sounding used for our parcel calculation. The behavior higher up is, however, not very sensitive to sounding variations (see the following subsection).

\section{2) ForCE BALANCE AND ROLE OF INITIAL MOMENTUM}

Because of strong entrainment, buoyancies are weaker in the new model; this plus the absence of friction cause inertia to be much more important. The lower buoyancies are consistent with error-corrected observations showing typical buoyancies of less than $1^{\circ} \mathrm{C}$ (about $0.03 \mathrm{~m} \mathrm{~s}^{-2}$ ) in cumulus congestus (Sinkevich and Lawson 2005) and with buoyancies calculated from the CRM output. The weaker damping (greater inertia) also seems more consistent with the weak relationship between buoyancy and ascent in simulated cumuli (Xu and Randall 2001) and the ability of temperatures above deep convective outflow to determine the outflow height (Chae and Sherwood 2010).

The dependence of the final cloud height on initial $W$ is shown in Fig. 12. For either the standard or new model, a vertical velocity of at least $7 \mathrm{~m} \mathrm{~s}^{-1}$ is needed to overcome the convective inhibition in this particular initial sounding. However, a similar calculation with a mean sounding from the western Pacific region (Fig. 13) only requires a $3 \mathrm{~m} \mathrm{~s}^{-1}$ initial velocity. Convective inhibition is highly variable and is easily modified by the convection itself, so it may be less important in realistic situations than in parcel calculations.

Once there is sufficient initial momentum to overcome convective inhibition, the depth of convection is insensitive to further increases in initial $W$ in the standard model, but the depth does show some sensitivity in the new model. This could prove to be relevant to convective triggering, for example as observed on frontal 


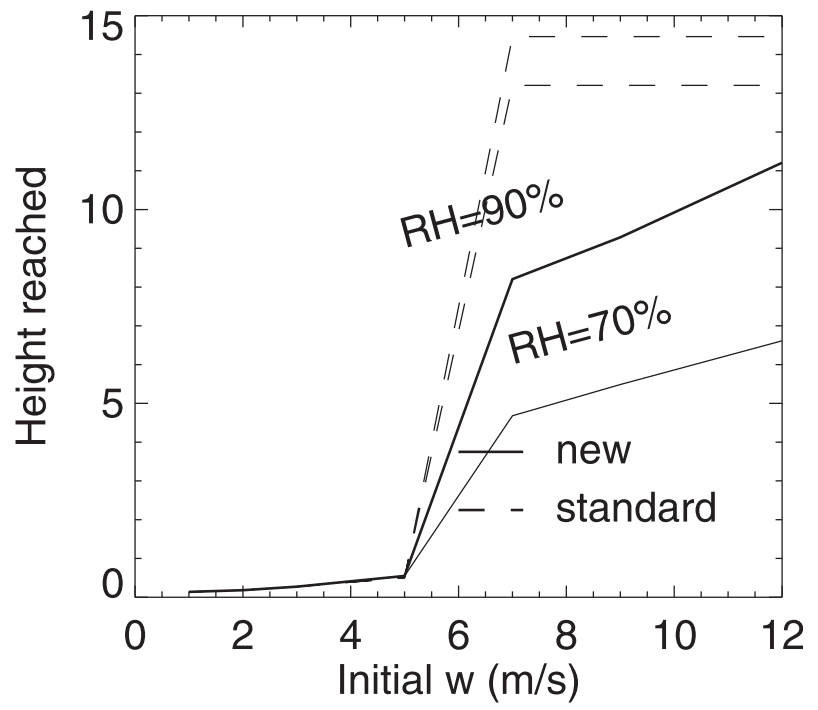

FIG. 12. Cloud height vs initial upward velocity for the standard and new models and for two values of ambient free-tropospheric relative humidity as indicated.

boundaries and topographic features (Weckwerth and Parsons 2006). We were unable to test this further in WRF due to ambiguity in identifying initial parcel conditions, but intend to pursue this further in subsequent work.

\section{3) Cloud Amount AND WATER CONTENT}

Cloud water content differs dramatically between the two calculations. Low or nonentraining parcels retain far too much condensed water, especially at high altitudes, which must be removed by immediate and highly efficient precipitation. The strong entrainment in the new model produces much lower water amounts, peaking at midtroposphere, roughly matching peak ice amounts in the WRF simulations and in previous studies ( $\mathrm{Xu}$ and Randall 2001).

Recall that our parcel is a heterogeneous collection of air with different histories, enabling a direct calculation of the cloudy fraction. In principle this could be taken as an indicator of cloud volume per unit ascending air volume. To test how well this works, we estimate the total volume of ascending air in the CRM as all regions of $w>2 \mathrm{~m} \mathrm{~s}^{-1}$, and the volume of ascending air in our parcel model as half the parcel volume (since roughly half the thermal vortex is rising at any given instant). We then compare the ratio of cloud to updraft volumes in the parcel model and in the CRM. The parcel model predicts this fraction to be very closely proportional to environmental relative humidity, which also happens in the CRM (Fig. 14). Standard parcel models would not predict this relationship: since the cloud and updraft are conceptually identical, the ratio of their volumes is

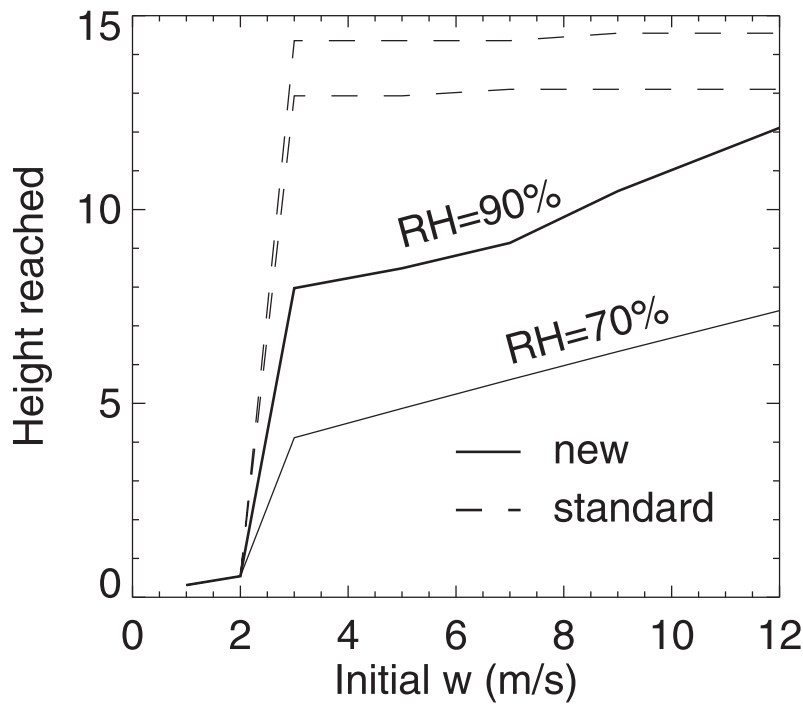

FIG. 13. As in Fig. 12, but with a mean sounding from the western Pacific region.

always unity. The slope of the relationship depends on entrainment rate, such that the agreement here further supports an effective rate of $\sim(2 \mathrm{~km})^{-1}$. Given the difficulties in measuring (or indeed defining) entrainment directly, this type of test could be a useful constraint on diagnosed entrainment rates.

\section{Discussion and conclusions}

We have delimited and tracked spherical regions centered on maximal local vertical velocities $w$, which approximately bound individual thermal vortices in numerical simulations; estimated their entrainment; and analyzed their vertical momentum budgets. Such thermals are stabilized by their internal vorticity and move as coherent blobs of air, except for entrainment and detrainment, as well known from laboratory studies. We find that the tracked $w$ maxima ascend at nearly constant velocities $W$, typically $3-5 \mathrm{~m} \mathrm{~s}^{-1}$, about $2-3$ times slower than $w$. From inspection of the data we judge that during the congestus or deepening phase, most of the strongest $w$ do coincide with vortex-like structures (albeit often highly distorted), and that the conclusions below apply generally in the vicinity of the strongest updrafts.

In diagnosing updraft characteristics, past large-eddy simulation (LES-CRM studies have typically followed a conditional-sampling approach based on vertical velocity, buoyancy, and/or cloud water criteria to establish control regions for analysis (e.g., de Roode and Bretherton 2003). While this may be adequate for some purposes, we argue that the conditionally sampled regions exclude portions of the true parcel (and 


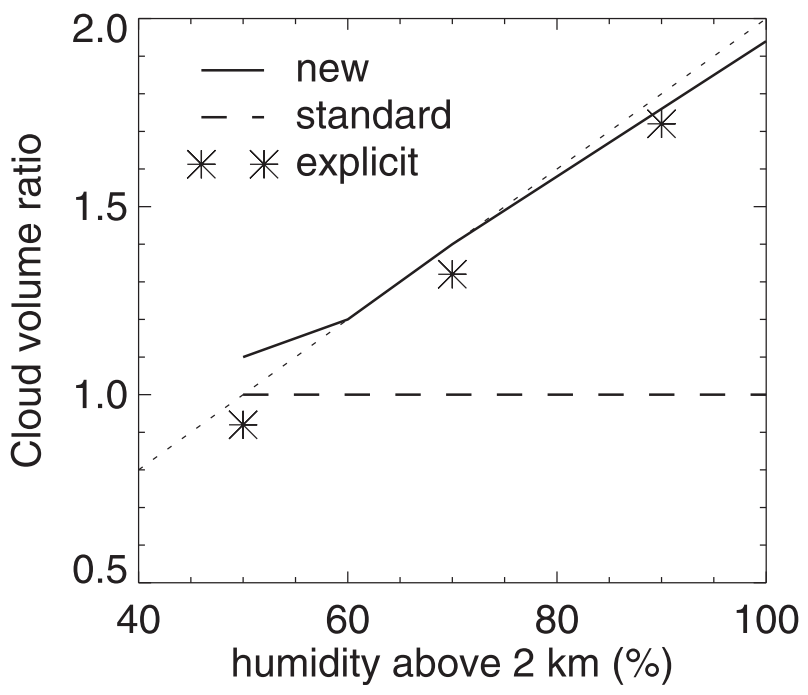

FIG. 14. Cloud volume per unit updraft volume below $8 \mathrm{~km}$ in an explicit cloud simulation (asterisks) and calculated by the new (solid line) and standard (dashed line) models vs environmental humidity. The dotted line shows the result if cloud fraction in the thermal equals relative humidity.

potentially include cloudy air that is no longer part of a rising parcel) and thereby may not be ideal for constraining dynamical assumptions in a parcel-based cloud model. By accounting for the entire thermal we obtain slower parcel ascents, and perhaps a simpler picture of parcel dynamics. The outer part of the thermal neglected in previous studies is probably similar to the region sometimes called the cloud shell that has been seen to interact with the updraft (Heus and Jonker 2008; Dawe and Austin 2011), but we assert that some of this air is dynamically part of the ascending air parcel rather than a separate downdraft (although separate downdrafts may also exist).

The relatively slow ascent rate $W$ of the thermals means they would require nearly an hour to reach the upper troposphere. This alone introduces a delay into the growth of convection that may help explain why observed convection deepens more slowly than that parameterized in global models. Moreover, multiple thermals must ascend through a growing cumulus as it penetrates into the upper troposphere, even in our simulations, which are forced in a relatively strong way by converging sea-breeze fronts. These factors add to those already considered in the literature for explaining the slowness of convective deepening (see Del Genio 2012).

Entrainment rates are high [e.g., $(2-3 \mathrm{~km})^{-1}$ ], but the drag associated with this mixing is very small and in most cases negative (i.e., accelerating the thermal). The momentum budget during much of the thermal lifetime accordingly consists of a near balance between buoyancy and nonhydrostatic forces. This supports results by de Roode et al. (2012), who find that drag forces associated with mixing are not as important as usually assumed, whereas the pressure gradient term is the dominant drag force on rising cumuli. We offer a physical hypothesis of why this anticipated drag is not occurring based on the structure of an idealized thermal (Fig. 7). A better understanding of the nonhydrostatic forces would be valuable but is deferred to later study.

Drag forces are implicitly assumed to dominate inertia in many deep convection schemes, but individual forces found here are comparable to, or smaller than, the ratio of thermal ascent rate to lifetime, indicating that thermals are not strongly damped. Moreover, it is uncertain how much of the nonhydrostatic resistance should be regarded as a passive drag and how much is better viewed as a dynamical offset to the buoyancy. Further work will examine this.

A simple parcel calculation shows that the treatment of drag could have important implications for cumulus parameterization. We find that a strongly entraining parcel could not ascend very far in a typical environment if this were accompanied by the momentum dilution traditionally assumed (although with other variations to the calculation this may be possible; Romps and Kuang 2010), even with no other drag forces, but that such ascent becomes much easier without momentum dilution.

Modelers are now well aware that these schemes are often insufficiently sensitive to midlevel moisture (e.g., Del Genio 2012). While stronger entrainment can fix this (e.g., Sahany et al. 2012), very large rates would be required to reproduce observed height sensitivity to moisture quantitatively (Sherwood et al. 2004), and such large rates should stifle deep convection under traditional assumptions. One solution is to assume that entrainment rates increase in dry environments, even though there is no fundamental justification for this, it is not suggested by regional simulations (Del Genio and Wu 2010), and it is not supported by our simulations. We suggest another possibility, which is that entrainment rates are always high during the formative stages of deep convection, making growth rates naturally sensitive to midlevel humidity (e.g., Holloway and Neelin 2009). Small buoyancy and drag forces further enhance the sensitivity from entrainment, because a given reduction in buoyancy from entrained dry air has a proportionally stronger effect on $W$.

Our findings for cumulus congestus may not apply to fully developed and/or more organized storms, which some studies suggest, for example, have lower entrainment (cf. Del Genio and Wu 2010). We find entrainment distances increase to as much as $5 \mathrm{~km}$ in the strongest simulated updrafts, and could increase further in simulations with strong large-scale forcing. This may be 
important in accounting for the high $\theta_{e}$ in the upper tropical troposphere, for example. But since all storms must first go through a growth phase, our results seem important even though they may not apply later. If entrainment only becomes weak after convection has fully developed, then it is not appropriate to use weakly entraining plumes to establish the degree of convective development in a model.

Our parcel calculations show that a high entrainment rate, near $(2 \mathrm{~km})^{-1}$, not only reproduces qualitatively the observed sensitivity of growth to midlevel moisture but also brings the predictions of some key cloud properties into quantitative alignment with the CRM results. First, predicted peak condensed water amounts become similar at all heights to the highest amounts predicted at those heights by the CRM. By contrast, with lower entrainment rates, the adiabatic water contents are much larger than those seen anywhere in the CRM. Second, the new parcel model correctly predicts the dependence of total cloud amount on ambient humidity seen in the CRM. This dependence is weak to nil with traditional assumptions.

While these changes are interesting, and suggest that lower friction could make some aspects of convective models more realistic, results could depend on many variables not examined here, and our model omits complications considered in real cumulus parameterizations. For example, the latter must simulate the effect of an ensemble of cumulus clouds rather than a single one. The various clouds are often assumed to entrain at different rates, and to occur in proportions needed to satisfy mass-flux constraints. Since the most weakly entraining plumes in such an ensemble penetrate the farthest, they may be required to account for the mass fluxes in the upper troposphere, which are ultimately demanded by radiative cooling near the tropopause. Thus, the structural assumptions of such schemes may make weakly or nonentraining drafts unavoidable. Accounting for slippery and/or detraining thermals could change this picture substantially, since strongly entraining parcels could provide mass fluxes even in the upper troposphere.

Some studies argue that entrainment rates should decrease linearly with height (e.g., Holloway and Neelin 2009), in contrast to our findings. Such inferences do rely heavily on the assumptions of the underlying plume model, which we question here. On the other hand, since the more-weakly entraining parcels would reach higher, a linear decrease could apply statistically within a parcel ensemble even if it did not apply to any individual air parcel, offering a possible reconciliation. Further work will be necessary to clarify what the real implications are of slippery thermals in an ensemble situation, or a parameterization.
Acknowledgments. This work was supported by the NSF Physical and Dynamical Meteorology program, Grant DYN078550. The computational component of this work was supported in part by the facilities and staff of the Yale University Faculty of Arts and Sciences High Performance Computing Center. SCS thanks S. Turner for helpful comments.

\section{REFERENCES}

Batchelor, G. K., 1967: An Introduction to Fluid Mechanics. Cambridge University Press, $615 \mathrm{pp}$.

Betts, A. K., 1982: Saturation point analysis of moist convective overturning. J. Atmos. Sci., 39, 1484-1505.

Blyth, A. M., W. A. Cooper, and J. B. Jensen, 1988: A study of the source of entrained air in Montana cumuli. J. Atmos. Sci., 45, 3944-3964.

— S. G. Lasher-Trapp, and W. A. Cooper, 2005: A study of thermals in cumulus clouds. Quart. J. Roy. Meteor. Soc., 131, $1171-1190$

Carpenter, R. L., K. K. Droegemeier, and A. M. Blyth, 1998: Entrainment and detrainment in numerically simulated cumulus congestus clouds. Part III: Parcel analysis. J. Atmos. Sci., 55, 3440-3455.

Chae, J. H., and S. C. Sherwood, 2010: Insights into cloud-top height and dynamics from the seasonal cycle of cloud-top heights observed by MISR in the west Pacific region. J. Atmos. Sci., 67, 248-261.

Chepfer, H., S. Bony, D. Winker, M. Chiriaco, J. L. Dufresne, and G. Sèze, 2008: Use of CALIPSO lidar observations to evaluate the cloudiness simulated by a climate model. Geophys. Res. Lett., 35, L15704, doi:10.1029/2008GL034207.

Chikira, M., and M. Sugiyama, 2010: A cumulus parameterization with state-dependent entrainment rate. Part I: Description and sensitivity to temperature and humidity profiles. J. Atmos. Sci., 67, 2171-2193.

Dabiri, J. O., and M. Gharib, 2004: Fluid entrainment by isolated vortex rings. J. Fluid Mech., 511, 311-331.

Damiani, R., G. Vali, and S. Haimov, 2006: The structure of thermals in cumulus from airborne dual-Doppler radar observations. J. Atmos. Sci., 63, 1432-1450.

Dawe, J. T., and P. H. Austin, 2011: The influence of the cloud shell on tracer budget measurements of les cloud entrainment. J. Atmos. Sci., 68, 2909-2920.

Del Genio, A. D., 2012: Representing the sensitivity of convective cloud systems to tropospheric humidity in general circulation models. Surv. Geophys., 33, 637-656, doi:10.1007/ s10712-011-9148-9.

, and J. B. Wu, 2010: The role of entrainment in the diurnal cycle of continental convection. J. Climate, 23, 2722-2738.

Derbyshire, S. H., I. Beau, P. Bechtold, J.-Y. Grandpeix, J.-M. Pirou, J.-L. Redelsperger, and P. Soares, 2004: Sensitivity of moist convection to environmental humidity. Quart. J. Roy. Meteor. Soc., 130, 3055-3079.

de Roode, S. R., and C. S. Bretherton, 2003: Mass-flux budgets of shallow cumulus clouds. J. Atmos. Sci., 60,137-151.

— A. P. Siebesma, H. J. J. Jonker, and Y. de Voogd, 2012: Parameterization of the vertical velocity equation for shallow cumulus clouds. Mon. Wea. Rev., 140, 2424-2436.

Emanuel, K. A., 1991: A scheme for representing cumulus convection in large-scale models. J. Atmos. Sci., 48, 2313-2335. 
Fierro, A. O., J. Simpson, M. A. LeMone, J. M. Straka, and B. F. Smull, 2009: On how hot towers fuel the Hadley cell: An observational and modeling study of line-organized convection in the equatorial trough from TOGA COARE. J. Atmos. Sci., 66, 2730-2746.

Fridlind, A. M., and Coauthors, 2004: Evidence for the predominance of mid-tropospheric aerosols as subtropical anvil cloud nuclei. Science, 304, 718-722.

Heus, T., and H. J. J. Jonker, 2008: Subsiding shells around shallow cumulus clouds. J. Atmos. Sci., 65, 1003-1018.

—, G. Van Dijk, H. J. J. Jonker, and H. E. A. Van den Akker, 2008: Mixing in shallow cumulus clouds studied by Lagrangian particle tracking. J. Atmos. Sci., 65, 2581-2597.

Hill, M. J. M., 1894: On a spherical vortex. Philos. Trans. Roy. Soc., 185, 213-245.

Holloway, C. E., and J. D. Neelin, 2009: Moisture vertical structure, column water vapor, and tropical deep convection. J. Atmos. Sci., 66, 1665-1683.

John, V. O., and B. J. Soden, 2006: Does convectively-detrained cloud ice enhance water vapor feedback? Geophys. Res. Lett., 33, L20701, doi:10.1029/2006GL027260.

Keenan, T. D., and R. E. Carbone, 1992: A preliminary morphology of precipitation systems in tropical northern Australia. Quart. J. Roy. Meteor. Soc., 118, 283-326.

Khairoutdinov, M., and D. Randall, 2006: High-resolution simulation of shallow-to-deep convection transition over land. J. Atmos. Sci., 63, 3421-3436.

Malkus, J. S., 1954: Some results of a trade cumulus cloud investigation. J. Meteor., 11, 220-237.

—, R. S. Scorer, F. H. Ludlam, and O. Björgum, 1953: Bubble theory of penetrative convection. Quart. J. Roy. Meteor. Soc., 79, 288-293.

Mapes, B. E., and R. B. Neale, 2011: Parameterizing convective organization to escape the entrainment dilemma. J. $A d v$. Model. Earth Sys., 3, M06004, 10.1029/2011MS000042.

Morton, B. R., G. Taylor, and J. S. Turner, 1956: Turbulent gravitational convection from maintained and instantaneous sources. Proc. Roy. Soc. London, 234A, 1-23.

Narasimha, R., S. S. Diwan, S. Duvvuri, K. R. Sreenivas, and G. S. Bhat, 2011: Laboratory simulations show diabatic heating drives cumulus-cloud evolution and entrainment. Proc. Natl. Acad. Sci. USA, 108, 16164-16169.

Paluch, I. R., and D. G. Baumgardner, 1989: Entrainment and finescale mixing in a continental convective cloud. J. Atmos. Sci., 46, 261-278.

Riehl, H., and J. S. Malkus, 1958: On the heat balance in the equatorial trough zone. Geophysica, 6, 503-537.

Robinson, F. J., S. C. Sherwood, D. Gerstle, C. Liu, and D. J. Kirshbaum, 2011: Exploring the land-ocean contrast in convective vigor using islands. J. Atmos. Sci., 68, 602-618.

Romps, D. M., 2010: A direct measure of entrainment. J. Atmos. Sci., 67, 1908-1927.

— exist in the upper tropical troposphere? J. Atmos. Sci., 67, 468-484.

Sahany, S., J. D. Neelin, K. Hales, and R. B. Neale, 2012: Temperature moisture dependence of the deep convective transition as a constraint on entrainment in climate models. J. Atmos. Sci., 69, 1340-1358.
Sánchez, O., D. J. Raymond, L. Libersky, and A. G. Petschek, 1989: The development of thermals from rest. J. Atmos. Sci., 46, 2280-2292.

Saunders, P. M., 1961: An observational study of cumulus. J. Meteor., 18, 451-467.

Schumann, U., and C. H. Moeng, 1991: Plume budgets in clear and cloudy convective boundary layers. J. Atmos. Sci., 48, 1758-1770.

Scorer, R. S., and F. H. Ludlam, 1953: Bubble theory of penetrative convection. Quart. J. Roy. Meteor. Soc., 79, 94-103.

— Weather, 11, 151-155.

Sherwood, S. C., P. Minnis, and M. McGill, 2004: Deep convective cloud top heights and their thermodynamic control during CRYSTAL-FACE. J. Geophys. Res., 109, D20119, doi:10.1029/ 2004JD004811.

—, R. Roca, T. M. Weckwerth, and N. G. Andronova, 2010: Tropospheric water vapor, convection, and climate. Rev. Geophys., 48, RG2001, doi:10.1029/2009RG000301.

Siebesma, A. P., and J. W. M. Cuijpers, 1995: Evaluation of parametric assumptions for shallow cumulus convection. J. Atmos. Sci., 52, 650-666.

Simpson, J., 1983: Cumulus clouds: Numerical models, observations, and entrainment. Mesoscale Meteorology, D. K. Lilly and T. Gal-Chen, Eds., D. Reidel, 413-445.

Sinkevich, A. A., and R. P. Lawson, 2005: A survey of temperature measurements in convective clouds. J. Appl. Meteor., 44, 1133-1145.

Stommel, H., 1947: Entrainment of air into a cumulus cloud. J. Meteor., 4, 91-94.

Turner, J. S., 1957: Buoyant vortex rings. Proc. Roy. Soc. London, 239A, 61-75.

_ 1964: The flow into an expanding spherical vortex. J. Fluid Mech., 18, 195-208.

_ 1972: Energy deficiency in self-preserving convective flows. J. Fluid Mech., 53, 217-226.

_ 1973: Buoyancy Effects in Fluids. Cambridge University Press, 367 pp.

Warner, J., 1970: On steady-state one-dimensional models of cumulus convection. J. Atmos. Sci., 27, 1035-1040.

Weckwerth, T., and D. B. Parsons, 2006: A review of convection initiation and motivation for IHOP_2002. Mon. Wea. Rev., 134, 5-22.

Winker, D. M., and Coauthors, 2010: The CALIPSO Mission: A global 3D view of aerosols and clouds. Bull. Amer. Meteor. Soc., 91, 1211-1229.

Xu, K. M., and D. A. Randall, 2001: Updraft and downdraft statistics of simulated tropical and midlatitude cumulus convection. J. Atmos. Sci., 58, 1630-1649.

Zhang, Y., S. A. Klein, J. Boyle, and G. G. Mace, 2010: Evaluation of tropical cloud and precipitation statistics of Community Atmosphere Model version 3 using CloudSat and CALIPSO data. J. Geophys. Res., 115, D12205, doi:10.1029/2009JD012006.

Zhao, M., and P. H. Austin, 2005: Life cycle of numerically simulated shallow cumulus clouds. Part II: Mixing dynamics. J. Atmos. Sci., 62, 1291-1310.

Zhou, L., R. B. Neale, M. Jochum, and R. Murtugudde, 2012: Improved Madden-Julian oscillations with improved physics: The impact of modified convection parameterizations. J. Climate, 25, 1116-1136. 